\title{
Combinatorial interpretation of Kolmogorov complexity
}

\author{
A. Romashchenko \\ Moscow State University \\ anromash@mccme.ru
}

\author{
A. Shen \\ Institute of Problems of \\ Information Transmission \\ shen@mccme.ru
}

\author{
N. Vereshchagin \\ Moscow State University \\ ver@mccme.ru
}

\begin{abstract}
Kolmogorov's very first paper on algorithmic information theory (Kolmogorov, Problemy peredachi infotmatsii 1(1) (1965), 3) was entitled "Three approaches to the definition of the quantity of information". These three approaches were called combinatorial, probabilistic and algorithmic. Trying to establish formal connections between combinatorial and algorithmic approaches, we prove that every linear inequality including Kolmogorov complexities could be translated into an equivalent combinatorial statement. (Note that the same linear inequalities are true for Kolmogorov complexities and Shannon entropy, see Hammer et al., (Proceedings of CCC'97, Ulm).) Entropy (complexity) proofs of combinatorial inequalities given in Llewellyn and Radhakrishnan (Personal Communication) and Hammer and Shen (Theory Comput. Syst. 31 (1998) 1) can be considered as special cases (and a natural starting points) for this translation.
\end{abstract}

Keywords: Kolmogorov complexity; Inequalities; Combinatorics

\section{Introduction and examples}

Kolmogorov complexity $K(x)$ of a binary string $x$ is defined as the length of shortest program that produces $x$. Complexity depends on the programming system, and we assume that programming system is optimal (complexity is minimal up to $O(1)$ additive term). Conditional complexity $K(x \mid y)$ is defined as the length of shortest program that produces $x$ given input $y$.

This approach was called "algorithmic" in [1]. Combinatorial approach was explained in the same paper as follows:

Consider a variable $x$ whose range is a finite set $X$ of cardinality $N$. One can say that the "entropy" of variable $x$ is equal to $H(x)=\log _{2} N$. When a specific value $x=a$ is fixed, we "eliminate" this entropy by providing $I=\log _{2} N$ bits of "information". For $k$ independent variables $x_{1}, \ldots, x_{k}$ whose range have cardinalities $N_{1}, \ldots, N_{k}$ we have $H\left(x_{1}, x_{2}, \ldots, x_{k}\right)=H\left(x_{1}\right)+H\left(x_{2}\right)+\ldots+$ $H\left(x_{k}\right)$.

And later:

Let $x$ and $y$ be variables (with ranges $X$ and $Y$ ) that are dependent in the following sense: not all pairs $x, y$ from $X \times Y$ are allowed as values. Let $U$ be the set of all allowed pairs. For any $a \in X$ we consider the set $Y_{a}$ of all $y$ such that $(a, y) \in U$. Now the conditional entropy can be naturally defined as follows: $H(y \mid a)=\log _{2} N\left(Y_{a}\right)$ where $N\left(Y_{a}\right)$ stands for the cardinality of $Y_{a}$.

There are some evident connections between combinatorial and algorithmic approaches. First, the set of all strings having complexity less than $n$ contains at most $2^{n}$ elements (since different strings correspond to different programs and the number of programs does not exceed $\left.1+2+\ldots+2^{n-1}\right)$. On the other hand, as Kolmogorov says, if a finite set $M$ with large cardinality $N$ can be defined by a program of a negligible length (compared to $\log _{2} N$ ), then almost all elements of $M$ have complexity close to $\log _{2} N$ [1]. 
Therefore the statement $K(x)<n$ can be informally translated into combinatorial language as $x$ belongs to a naturally defined set of cardinality about $2^{n}$.

In this section we give several examples showing a similarity between combinatorial and algorithmic approaches. In the next section we formulate three theorems that provide combinatorial translations for linear inequalities involving Kolmogorov complexities. All logarithms are binary: $\log u$ stands for $\log _{2} u$.

Our first example is the inequality

$$
K(x, y) \leq K(x)+K(y)+O(\log (K(x)+K(y)))
$$

Here $x$ and $y$ are binary strings; $K(x, y)$ denotes the complexity of pair $(x, y)$ defined as complexity of the string $[x, y]$ for a computable encoding $x, y \mapsto[x, y]$ (different encodings give different complexities, but the difference is $O(1)$ ).

The combinatorial counterpart of this inequality is the following statement: Let $A$ be a subset of the product $X \times$ $Y$ of two finite sets $X$ and $Y$. Then

$$
\# A \leq \# \pi_{X}(A) \cdot \# \pi_{Y}(A)
$$

where \# stands for cardinality, $\pi_{X}$ and $\pi_{Y}$ are projections (e.g., $\pi_{X}(A)=\{x \in A \mid \exists y\langle x, y\rangle \in A\}$ ).

The similarity is straightforward: take logarithms and recall that "combinatorial entropy" is the logarithm of cardinality of range. If a pair of variables $x, y$ ranges over $A \subset X \times Y$, then $x$ ranges over $\pi_{X}(A)$ and $y$ ranges over $\pi_{Y}(A)$.

Now consider a stronger inequality

$$
\begin{aligned}
& \quad K(x, y) \leq \\
& \quad \leq K(x)+K(y \mid x)+O(\log (K(x)+K(y)))
\end{aligned}
$$

(Let us note that all inequalities for complexities are considered up to $O(\log m)$-term where $m$ is the sum of complexities of all strings involved; we omit $O(\log m)$-terms (and $O(1)$-terms) in the sequel.)

Inequality (3) is stronger than (1) since $K(y \mid x) \leq$ $K(y)$.

Recalling Kolmogorov's explanation of the combinatorial meaning of conditional entropy, we come to the following inequality:

$$
\# A \leq \# \pi_{X}(A) \cdot\left[\max _{x \in X} \# A_{x}\right]
$$

where $A_{x}$ stands for the set $\{y \mid\langle x, y\rangle \in A\}$. Note that the inequality (4) is stronger than (2) since \# $A_{x} \leq$ $\# \pi_{Y}(A)$ for any $x \in A$.

The next example involves three variables and is considered in detail in [2]. The inequality

$$
2 K(x, y, z) \leq K(x, y)+K(x, z)+K(y, z)
$$

is true (up to logarithmic terms) for any three strings $x, y, z$. Its combinatorial counterpart says that

$$
(\# A)^{2} \leq \# \pi_{X Y}(A) \cdot \# \pi_{X Z}(A) \cdot \# \pi_{Y Z}(A)
$$

for any subset $A$ of the Cartesian product $X \times Y \times Z$ of three finite sets $X, Y$ and $Z$. (Here $\pi_{X Y}$ stands for the projection of $X \times Y \times Z$ onto $X \times Y$ etc.)

This inequality also can be strengthened by replacing unconditional complexity by conditional one:

$$
2 K(x, y, z) \leq K(x, y)+K(x, z)+K(y, z \mid x)
$$

The combinatorial counterpart is

$$
(\# A)^{2} \leq \# \pi_{X Y}(A) \cdot \# \pi_{X Z}(A) \cdot\left[\max _{x \in X} \# A_{x}\right]
$$

where $A_{x}=\{\langle y, z\rangle \mid\langle x, y, z\rangle \in A\}$

All four examples given above follow the same pattern and are covered by theorem 1 below; it says that combinatorial statement is true if and only if the corresponding inequality holds.

A more subtle example is provided by an inequality

$$
K(x)+K(y \mid x) \leq K(x, y)
$$

where, as usual, logarithmic terms are omitted. (This inequality is a reversed form of (3), so in fact inequality (3) is an equality.) What is the corresponding combinatorial statement? One could try

$$
\# \pi_{X}(A) \cdot\left[\max _{x \in X} \# A_{x}\right] \leq \# A,
$$

but this statement is false for evident reasons (consider $A$ that has large $A_{x}$ for some $x$ and small $A_{x}$ for many other $x$ 's). However, one can find a true statement which looks parallel to (9). Here it is:

Let $X$ and $Y$ be two finite sets and let $A$ be a subset of $X \times Y$. Let $u$ and $v$ be two integers such that $u v \geq \# A$. Then $A$ can be partitioned into $A=U \cup V$ with $\# \pi_{X}(U) \leq u$ and $\max _{x \in X} \# V_{x} \leq$ $v$. 
To prove (10) consider the set $T$ of all $x \in X$ such that $\# A_{x}>v$. This set contains at most $u$ elements (otherwise $\# A>u v$ ). Now let $U$ be the set of all $\langle x, y\rangle \in A$ such that $x \in T$ and let $V$ be the remaining part of $A$. Then $\pi_{X}(U)=T$ and $\# \pi_{X}(U) \leq u$; on the other hand, $\# V_{x}$ is zero for $x \in T$ and does not exceed $v$ for $x \notin T$, therefore, $\max _{x \in X} \# V_{x} \leq v$.

In fact, the statement (10) can be used as an intermediate step in the proof of (9).

Our last example is the so-called "basic inequality" from [4], i.e., the inequality

$$
K(x)+K(x, y, z) \leq K(x, y)+K(x, z)
$$

This inequality follows from the inequality $K(y, z \mid x) \leq$ $K(y \mid x)+K(z \mid x)$ (which is a "conditional version" of (1)) using the equalities $K(x, y)=K(y \mid x)+K(x)$, $K(x, z)=K(z \mid x)+K(x)$ and $K(x, y, z)=K(y, z \mid x)+$ $K(x)$; all three equalities mentioned follow from (3) and (9).

Inequality (11) corresponds to the following combinatorial statement:

Let $X, Y$ and $Z$ be three finite sets and let $A$ be a subset of $X \times Y \times Z$. Let $l$ and $v$ be two integers such that $l v \geq \# \pi_{X Y}(A) \cdot \# \pi_{X Z}(A)$. Then $A$ can be partitioned into $A=U \cup V$ with $\# \pi_{X}(U) \leq l$ and $\# V \leq v$.

This statement can be proved as follows. For each $x \in X$ consider the set

$$
A_{x}=\{\langle y, z\rangle \mid\langle x, y, z\rangle \in A\}
$$

The set $X$ can be linearly ordered in such a way that $\# A_{x}$ decreases as $x$ increases. Consider $l$ first elements of $X$ in this ordering. Corresponding triples form the set $U$; the remaining part of $A$ goes to $V$. (It is easy to see that this choice is optimal; we want to make $\# V$ smaller, so we include large $A_{x}$ into $U$.) The construction guarantees that $\# \pi_{X}(U) \leq l$. It remains to prove that $\# V \leq v$.

Let $S_{1}$ and $S_{2}$ be the cardinalities of $\pi_{X Y}(A)$ and $\pi_{X Z}(A)$. Let us prove first that all $A_{x}$ outside $U$ have cardinalities at most $S_{1} S_{2} / l^{2}$. Let $p(x)$ and $q(x)$ be the cardinalities of projections of $A_{x}$ onto $Y$ and $Z$. Then $\sum_{x} p(x)=S_{1}$ and $\sum_{x} q(x)=S_{2}$. Therefore, the average value of $p(x)$ for $l$ first values of $x$ (corresponding to the set $U$ ) does not exceed $S_{1} / l$; the average value of $q(x)$ for $l$ first values of $x$ does not exceed $S_{2} / l$. Using Cauchy inequality, we conclude that the geometric mean of $l$ first values of $p(x)$ [of $q(x)$ ] does not exceed $S_{1} / l$ [resp. $\left.S_{2} / l\right]$. Therefore, the geometric mean of the product $p(x) q(x)$ does not exceed $S_{1} S_{2} / l^{2}$, and the minimal value of $p(x) q(x)$ does not exceed $S_{1} S_{2} / l^{2}$. Since $\# A_{x} \leq p(x) q(x)$, the minimal value of \# $A_{x}$ in $U$ (and all the values outside $U$ ) does not exceed $S_{1} S_{2} / l^{2}$.

Now we know that $\# V_{x} \leq S_{1} S_{2} / l^{2}$ for all $x$ (here $V_{x}=\varnothing$ for $l$ first values of $x$ and $V_{x}=A_{x}$ for remaining $x$ ). It remains to apply the inequality (8) to get the desired result:

$$
\# V \leq \sqrt{S_{1} \cdot S_{2} \cdot \frac{S_{1} S_{2}}{l^{2}}}=\frac{S_{1} S_{2}}{l} \leq v .
$$

The statement (12) is proved.

\section{Linear inequalities}

We hope that the examples above make clear the correspondence between complexity inequalities and combinatorial statements. However, let us give the exact definitions for the general case.

We consider linear inequalities involving strings $x_{1}, \ldots, x_{s}$. (The number $s$ of strings is a constant.) For any set $I \subset\{1, \ldots, s\}$ containing elements $i_{1}, \ldots, i_{m}$ we denote by $x_{I}$ the tuple $\left\langle x_{i_{1}}, \ldots, x_{i_{m}}\right\rangle$. Its complexity (defined in a natural way using encodings) is denoted by $K\left(x_{I}\right)$. For example, the basic inequality (11) can be written in this notation as

$$
K\left(x_{\{1\}}\right)+K\left(x_{\{1,2,3\}}\right) \leq K\left(x_{\{1,2\}}\right)+K\left(x_{\{1,3\}}\right)
$$

The general form of the linear inequality involving complexities of strings $x_{1}, \ldots, x_{s}$ and their combinations is

$$
\sum_{I} \lambda_{I} K\left(x_{I}\right) \geq 0 .
$$

The general form of an inequality involving conditional complexities is

$$
\sum_{I \cap J=\varnothing} \lambda_{I, J} K\left(x_{I} \mid x_{J}\right) \geq 0 .
$$


We assume that $I \cap J=\varnothing$ since $K\left(x_{I} \mid x_{J}\right)=$ $K\left(x_{I \backslash J} \mid x_{J}\right)$.

Now we need to introduce the notation for combinatorial statements. Let $X_{1}, \ldots, X_{s}$ be sets. For each $I \subset$ $\{1, \ldots, s\}$ we consider a projection function $\pi_{I}$ that maps $X_{1} \times \ldots \times X_{s}$ onto $\prod_{i \in I} X_{i}$. For any $A \subset X_{1} \times \cdots \times X_{s}$ by $\pi_{I}(A)$ we denote the image of $A$ under this projection; $n_{I}(A)=\# \pi_{I}(A)$ is its cardinality. (According to Kolmogorov, $\log n_{I}(A)$ can be considered as "combinatorial entropy" of projection $x_{I}$ if $x$ ranges over $A$.)

Conditional combinatorial entropy can be defined in a similar way. Let $I$ and $J$ be disjoint subsets of the index set $\{1, \ldots, s\}$. For any $a \in A$ consider a section of $A$ going through $a$ and having all $J$-coordinates fixed; consider $I$-projection of this section. Cardinality of this projection depends on $a$; let $n_{I \mid J}(A)$ be the maximal cardinality. Reformulation: fix $J$-coordinates of a variable $a \in A$ and consider the set of all possible values of $I$-coordinates. (This set depends on the values of $J$-coordinates.) Maximal cardinality of this set is denoted by $n_{I \mid J}(A)$.

The connection between combinatorial entropy and Kolmogorov complexity can be informally described as follows. Let $A$ be a set whose elements are tuples of strings $\left\langle x_{1}, \ldots, x_{s}\right\rangle$. Assume that Kolmogorov complexity of $A$ is small. Then the maximal value of $K\left(x_{I} \mid x_{J}\right)$ over all $\left\langle x_{1}, \ldots, x_{s}\right\rangle \in A$ is close to $\log n_{I \mid J}(A)$. Indeed, to specify $x_{I}$ when $x_{J}$ is known, we need $\log N$ bits, where $N$ is the number of possible values of $x_{I}$ when $x_{J}$ is known. This simple observation (refined in an appropriate way) is the main point of the proofs given below.

Our first theorem considers the case when only one coefficient $\lambda_{I, J}$ is negative. In other words, we consider inequality of type

$$
K\left(x_{I_{0}} \mid x_{J_{0}}\right) \leq \sum_{I, J} \lambda_{I, J} K\left(x_{I} \mid x_{J}\right)
$$

where summation ranges over pairs of disjoint sets different from $\left(I_{0}, J_{0}\right)$ and all $\lambda_{I, J}$ are non-negative.

Theorem 1 The inequality (14) is valid for all binary strings $x_{1}, \ldots, x_{s}$ (up to $O\left(\log \left(K\left(x_{1}\right)+\ldots+K\left(x_{s}\right)\right)\right)$ term) if and only if

$$
n_{I_{0} \mid J_{0}}(A) \leq \prod_{I, J}\left[n_{I \mid J}(A)\right]^{\lambda_{I, J}}
$$

for any subset $A \subset X_{1} \times \ldots \times X_{s}\left(X_{1}, \ldots, X_{s}\right.$ are arbitrary finite sets).

This theorem can be applied to the examples given above: it says that (1) is equivalent to (2), that (3) is equivalent to (4), that (5) is equivalent to (6), and that (7) is equivalent to (8). A special case of this theorem (inequalities (5) and (6)) was considered in [2]. Other special cases of this theorem and theorem 2 below are considered in [5]; in this paper Shannon entropy is used instead of Kolmogorov complexity and all $X_{i}$ have two elements (this restriction is not essential).

Proof. Let us prove (15) $\Rightarrow$ (14) first. Let $x_{1}, \ldots, x_{s}$ be arbitrary strings and $k_{I \mid J}=K\left(x_{I} \mid x_{J}\right)$. Consider the set $A$ of all tuples $y=\left\langle y_{1}, \ldots, y_{s}\right\rangle$ such that $K\left(y_{I} \mid y_{J}\right) \leq$ $k_{I \mid J}$ for all $(I, J) \neq\left(I_{0}, J_{0}\right)$. We want to apply (15) to $A$. It is easy to see that $\log n_{I \mid J}(A) \leq k_{I \mid J}+O(1)$. Indeed, if $y_{J}$ is fixed, only $2 \cdot 2^{k_{I \mid J}}$ values of $y_{I}$ are possible, since these values are obtained from $y_{J}$ by programs of length at most $k_{I \mid J}$. Applying (15) to $A$, we conclude that $\log n_{I_{0} \mid J_{0}}(A) \leq \sum \lambda_{I, J} k_{I \mid J}+O(1)$. Note also that the set $A$ can be enumerated effectively provided all $k_{I \mid J}$ are given (we need $O\left(\log \left(K\left(x_{1}\right)+\ldots+K\left(x_{s}\right)\right)\right)$ bits to specify all $\left.k_{I \mid J}\right)$. Now we see that $I_{0}$-coordinates of any element $y$ of $A$ are determined by $J_{0}$-coordinates of $y$ and its ordinal number in the enumeration of all $A$-elements having given $J_{0}$-coordinates. This number has $\log n_{I_{0} \mid J_{0}}$ bits, so we get (14).

Formally speaking, there is an error in this argument: we cannot apply (15) to $A$ directly, since $A$ can be infinite. However, we can apply (15) to all finite subsets of $A$ : if $n_{I_{0} \mid J_{0}}\left(A^{\prime}\right) \leq c$ for all finite $A^{\prime} \subset A$, then $n_{I_{0} \mid J_{0}}(A) \leq c$.

Now let us prove (14) $\Rightarrow(15)$. This proof is given in [2] for the special case of inequalities (5) and (6). It uses some trick: to get rid of logarithmic terms, we consider a sequence of elements of $A$ instead of one element.

We may assume that $X_{1}, \ldots, X_{s}$ are sets of binary strings. Let $M$ be a natural number. Let $\boldsymbol{y}=y^{1}, \ldots, y^{M}$ be a sequence of arbitrary elements of $A$. Each $y^{i}$ is a sequence of strings $y_{1}^{i}, \ldots, y_{s}^{i}$, so $\boldsymbol{y}$ can be considered as a matrix with $M$ rows and $s$ columns. For any set $I \subset\{1, \ldots, s\}$ we denote the sequence $y_{I}^{1}, \ldots, y_{I}^{M}$ by $\boldsymbol{y}_{I}$. (To get $\boldsymbol{y}_{I}$ from $\boldsymbol{y}$ we consider only columns of the matrix whose numbers belong to $I$.)

Now we apply the inequality (14) to the columns of the matrix. For any disjoint sets $I, J \subset\{1, \ldots, s\}$ the 
complexity $K\left(\boldsymbol{y}_{I} \mid \boldsymbol{y}_{J}\right)$ does not exceed $M \log n_{I \mid J}(A)+$ $O(\log M)$ where the constant in $O$-notation depends on $A$ but not on $M$. Indeed, to specify $\boldsymbol{y}_{I}$ when $\boldsymbol{y}_{J}$ is known we need (for each row $i$ ) to use $\log n_{I \mid J}$ bits for the ordinal number of $y_{I}^{i}$ in the set of all possibilities (for given $y_{J}^{i}$ ).

Therefore, for any $y^{1}, \ldots, y^{M} \in A$ we have

$$
K\left(\boldsymbol{y}_{I_{0}} \mid \boldsymbol{y}_{J_{0}}\right) \leq M \sum \lambda_{I, J} \log n_{I \mid J}(A)+O(\log M) .
$$

Now we want to get an upper bound for $n_{I_{0} \mid J_{0}}(A)$. Fix some value of $J_{0}$-coordinates. We want to get an upper bound for the number $N$ of possible values of $I_{0}$ coordinates compatible with fixed $J_{0}$-coordinates. Consider an arbitrary matrix $\boldsymbol{y}$ where all rows have given $J_{0}$ coordinates. Since $J_{0}$-coordinates are fixed, $K\left(\boldsymbol{y}_{J_{0}}\right)=$ $O(\log M)$ and $K\left(\boldsymbol{y}_{I_{0}}\right) \leq M \sum \lambda_{I, J} \log n_{I \mid J}(A)+$ $O(\log M)$. On the other hand, there are still $N^{M}$ possible values of $\boldsymbol{y}_{I_{0}}$, and all of them have bounded complexity, therefore

$$
\begin{aligned}
\log \left(N^{M}\right) & =M \log N \leq \\
& \leq M \sum \lambda_{I, J} \log n_{I \mid J}(A)+O(\log M) .
\end{aligned}
$$

Since $\log M / M \rightarrow 0$ as $M \rightarrow \infty$, we get the required upper bound for $N$.

Theorem 1 is proved.

Let us consider a special case of (14) when no conditional complexities are involved:

$$
K\left(x_{1}, \ldots, x_{s}\right) \leq \sum \lambda_{I} K\left(x_{I}\right)
$$

Here $\lambda_{I}$ are non-negative reals (for all $I \subsetneq\{1, \ldots, s\}$ ).

Theorem 2 The inequality (16) is true for all $x_{1}, \ldots, x_{s}$ (up to a logarithmic term) if and only if for any $j=$ $1, \ldots, s$ the sum of coefficients $\lambda_{I}$ for all I containing $j$ is at least 1.

Proof. Let $x_{i}$ be empty strings for all $i \neq j$. Then the inequality (16) can be rewritten as $K\left(x_{j}\right) \leq \sum \lambda_{I} K\left(x_{j}\right)$ where the sum is taken over all $I$ containing $j$. Therefore, if (16) is true for all strings, the sum of coefficients $\lambda_{I}$ is at least 1.

On the other hands, if all these sums are at least 1 , we can prove (16) as follows. Using (3) and (9), we rewrite

$$
\begin{aligned}
& K\left(x_{1}, \ldots, x_{s}\right) \text { as } \\
& K\left(x_{1}\right)+K\left(x_{2} \mid x_{1}\right)+K\left(x_{3} \mid x_{1}, x_{2}\right)+\ldots \\
& \quad \ldots+K\left(x_{s} \mid x_{1}, \ldots, x_{s-1}\right)
\end{aligned}
$$

and rewrite complexities in the right-hand side in the same way (using the same order of indices). For example, the term $K\left(x_{1}, x_{3}\right)$ in the right-hand side becomes $K\left(x_{1}\right)+$ $K\left(x_{3} \mid x_{1}\right)$. We then add omitted conditions in the righthand side (e.g., replace $K\left(x_{3} \mid x_{1}\right)$ by $K\left(x_{3} \mid x_{1}, x_{2}\right)$ ) and get a stronger inequality; this stronger inequality is valid according to our assumption (sum of coefficients for each $K\left(x_{i} \mid x_{1}, \ldots, x_{i-1}\right)$ is at least 1$)$.

Theorem 2 is proved.

This argument shows also that any valid inequality of type (16) is a positive linear combination of basic inequalities in the sense of [4].

Now we return to the general case and consider inequalities of type $\sum \lambda_{I, J} K\left(x_{I} \mid x_{J}\right) \geq 0$ where several coefficients may be negative. It is convenient to separate positive and negative coefficients and consider inequalities of type

$$
\sum_{(I, J) \in \mathcal{A}} \alpha_{I, J} K\left(x_{I} \mid x_{J}\right) \leq \sum_{(I, J) \in \mathcal{B}} \beta_{I, J} K\left(x_{I} \mid x_{J}\right)
$$

where all $\alpha_{I, J}$ and $\beta_{I, J}$ are positive and $\mathcal{A}, \mathcal{B}$ are disjoint sets of pairs of disjoint subsets of $\{1, \ldots, s\}$.

The following theorem gives a combinatorial statement that is equivalent to (17). Unfortunately, this condition is more complicated than one could expect looking at the relations between (9) and (10) or between (11) and (12). It includes a polynomial factor that corresponds to additive logarithmic term in the inequality about complexities.

Notation: $\mathbb{B}^{n}$ is a set of all binary strings of length $n$.

Theorem 3 The inequality (17) is valid for given coefficients $\alpha_{I, J}$ and $\beta_{I, J}$ and for any strings $x_{1}, \ldots, x_{s}$ (up to a logarithmic term) if and only if the following combina- 
torial statement is true:

there exists a constant $c$ such that for any $n$, for any set $A \subset\left(\mathbb{B}^{n}\right)^{s}$ and for any integers $a_{I, J}$ such that

$$
\prod_{(I, J) \in \mathcal{B}}\left[n_{I \mid J}(A)\right]^{\beta_{I, J}} \leq \prod_{(I, J) \in \mathcal{A}} a_{I, J}^{\alpha_{I, J}}
$$

the set $A$ can be covered by sets $U_{I, J}$ (for $(I, J) \in \mathcal{A})$ such that

$$
n_{I \mid J}\left(U_{I, J}\right) \leq a_{I, J} \cdot n^{c}
$$

Before proving this theorem, let us look at the combinatorial translation for the basic inequality (11): there exists a constant $c$ such that for all $n$, for any set $A \subset X \times Y \times Z$ (where $X=Y=Z=\mathbb{B}^{n}$ ) and for any $l$ and $v$ such that $\# \pi_{X Y}(A) \# \pi_{X Z}(A) \leq l v$ there exist $U$ and $V$ such that $A \subset U \cup V, \# \pi_{X}(U) \leq l n^{c}$ and $\# V \leq v n^{c}$. We see that the only difference between this statement and (12) is the factor $n^{c}$. (It seems quite possible that theorem 3 remains true without this factor. However, this factor is needed in our proof.)

Proof of theorem 3. Assume that the inequality (17) is valid up to a logarithmic term $O\left(\log \left(K\left(x_{1}\right)+\ldots+\right.\right.$ $\left.K\left(x_{s}\right)\right)$ ). We want to prove (18). For a given $n$ and given $A$ there exists some constant $c(n, A)$ that makes the statement (18) true (for all values of $a_{I, J}$ ). This is evident; what we need to prove is that the same constant works for all $n$ and all $A$. For a given $n$ consider the "worst-case" set $A_{n}$ and values of $a_{I, J}$ that require maximal constant. The set $A_{n}$ can be effectively found (try all possibilities; it is a very long, but finite, process). Therefore, complexity of $A_{n}$ is $O(\log n)$. For any $x \in A_{n}$ and for any disjoint $I, J \subset\{1, \ldots, s\}$ we have $K\left(x_{I} \mid x_{J}\right) \leq \log n_{I \mid J}\left(A_{n}\right)+O(\log n)$ (to specify $x_{I}$ when $x_{J}$ is fixed we need to specify $A_{n}$ and the ordinal number of $x_{I}$ ). Therefore, if numbers $a_{I, J}$ satisfy the inequality

$$
\prod_{(I, J) \in \mathcal{B}}\left[n_{I \mid J}\left(A_{n}\right)\right]^{\beta_{I, J}} \leq \prod_{(I, J) \in \mathcal{A}} a_{I, J}^{\alpha_{I, J}}
$$

then

$$
\begin{aligned}
& \sum_{(I, J) \in \mathcal{B}} \beta_{I, J} K\left(x_{I} \mid x_{J}\right) \leq \\
& \leq \sum_{(I, J) \in \mathcal{B}} \beta_{I, J} \log n_{I \mid J}(A)+O(\log n) \leq \\
& \quad \leq \sum_{(I, J) \in \mathcal{A}} \alpha_{I, J} \log a_{I, J}+O(\log n)
\end{aligned}
$$

Combining this inequality with (17), we conclude that

$\sum_{(I, J) \in \mathcal{A}} \alpha_{I, J} K\left(x_{I} \mid x_{J}\right) \leq \sum_{(I, J) \in \mathcal{A}} \alpha_{I, J} \log a_{I, J}+C \log n$

for any $x \in A_{n}$ and for some fixed $C$ (not depending on $n$ ). Therefore, if $x \in A_{n}$, then

$$
\alpha_{I, J} K\left(x_{I} \mid x_{J}\right) \leq \alpha_{I, J} \log a_{I, J}+\frac{C}{\# \mathcal{A}} \log n
$$

for at least one $(I, J) \in \mathcal{A}$. In other terms, sets

$$
U_{I, J}=\left\{x \mid K\left(x_{I} \mid x_{J}\right) \leq \log a_{I, J}+\frac{C}{\alpha_{I, J} \# \mathcal{A}} \log n\right\}
$$

cover $A$. And $\log n_{I \mid J}\left(U_{I, J}\right) \leq \log a_{I, J}+c \log n$ for some constant $c$ that does not depend on $n$. Since $A_{n}$ is the "worst-case" set by our assumption, we conclude that $c\left(n, A_{n}\right)$ is bounded by a constant not depending on $n$, and (18) is true.

To prove the second part of the theorem, assume that the statement (18) is true. We need to prove (17) for arbitrary tuple $x=\left\langle x_{1}, \ldots, x_{s}\right\rangle$. To do that, we "generalize" $x$ and include it in the set $A$ of tuples of strings that have "similar complexity behavior". Then we apply the statement (18) to $A$.

Formally $A$ is defined as the set of all tuples $y=$ $\left\langle y_{1}, \ldots, y_{s}\right\rangle$ such that $K\left(y_{I} \mid y_{J}\right) \leq K\left(x_{I} \mid x_{J}\right)$ for any disjoint sets $I, J \subset\{1, \ldots, s\}$. (This set was already used in the proof of theorem 1.) The set $A$ is not empty since it contains $x$. Moreover, $\log \# A$ is close to $K\left(x_{1}, \ldots, x_{s}\right)$. Indeed, $\log \# A$ cannot be significantly larger than $K\left(x_{1}, \ldots, x_{s}\right)$ because all $y \in A$ have complexity not exceeding $K\left(x_{1}, \ldots, x_{s}\right)$. On the other hand, $A$ can be enumerated by a program that has logarithmic (in $K\left(x_{1}\right)+\cdots+K\left(x_{s}\right)$ ) length (we need to specify all 
complexity bounds; number of these bounds is exponential in $s$, but $s$ is considered as a constant). Therefore, complexity of any $y \in A$ (including $x$ ) does not exceed significantly $\log \# A$, so $\log \# A$ cannot be significantly less than $K\left(x_{1}, \ldots, x_{s}\right)$.

The same argument shows that for any $(I, J)$ the number $\log n_{I \mid J}\left(A_{I, J}\right)$ differs from $K\left(x_{I} \mid x_{J}\right)$ at most by $O\left(\log \left(K\left(x_{1}\right)+\ldots+K\left(x_{s}\right)\right)\right)$.

To apply the statement (18) to $A$ we need to choose some value of $n$. Let $n$ be equal to $K\left(x_{1}\right)+\ldots+K\left(x_{s}\right)+$ 1. Using this value, we cannot apply (18) directly: an element $y=\left\langle y_{1}, \ldots, y_{s}\right\rangle \in A$ can contain very long $y_{i}$. However, the purely combinatorial nature of (18) allows us to rename all $y_{i}$. There is at most $2^{n}$ of them (since all $y$ 's have complexity less than $n$ ), and they can be replaced by strings of length $n$.

Now suppose that (in contradiction with (17))

$$
\begin{aligned}
\sum_{(I, J) \in \mathcal{B}} \beta_{I, J} K\left(x_{I} \mid x_{J}\right)< & \\
& <\sum_{(I, J) \in \mathcal{A}} \alpha_{I, J} K\left(x_{I} \mid x_{J}\right)-C \log n
\end{aligned}
$$

for some constant $C$ (to be fixed later).

Choose numbers $a_{I, J}^{\prime}$ such that

$$
\log a_{I, J}^{\prime}=K\left(x_{I} \mid x_{J}\right)-\frac{C}{\# \mathcal{A}} \log n
$$

Note that $a_{I, J}^{\prime}$ defined by this formula are not integers. Let $a_{I, J}$ be $\left\lceil a_{I, J}^{\prime}\right\rceil$. Then

$$
\log a_{I, J}=K\left(x_{I} \mid x_{J}\right)-\frac{C}{\# \mathcal{A}} \log n+O(1) .
$$

We have

$$
\sum_{(I, J) \in \mathcal{B}} \beta_{I, J} K\left(x_{I} \mid x_{J}\right)<\sum_{(I, J) \in \mathcal{A}} \alpha_{I, J} \log a_{I, J}
$$

i.e.,

$$
\prod_{(I, J) \in \mathcal{B}} K\left(x_{I} \mid x_{J}\right)^{\beta_{I, J}} \leq \prod_{(I, J) \in \mathcal{A}}\left[a_{I, J}\right]^{\alpha_{I, J}}
$$

Then by (18) the set $A$ can be covered by sets $U_{I, J}$ such that

$$
n_{I \mid J}\left(U_{I, J}\right) \leq a_{I, J} \cdot n^{c}
$$

For any $(I, J)$

$$
\begin{aligned}
& \log \# U_{I, J} \leq \log n_{J}\left(U_{I, J}\right)+\log n_{I \mid J}\left(U_{I, J}\right) \\
& \leq K\left(x_{J}\right)+\log a_{I, J}+c \log n \\
& \leq K\left(x_{J}\right)+K\left(x_{I} \mid x_{J}\right)-\frac{C}{\# \mathcal{A}} \log n+c \log n \\
& \leq K(x)+O(\log n)-\frac{C}{\# \mathcal{A}} \log n+c \log n .
\end{aligned}
$$

Since $A \subseteq \bigcup_{I, J} U_{I, J}$, and the number of pairs $(I, J)$ is a constant (for a fixed $s$ ), we have

$\log \# A \leq K(x)+O(\log n)-\frac{C}{\# \mathcal{A}} \log n+c \log n+O(1)$

Because $\log \# A$ cannot be significantly less then $K\left(x_{1}, \ldots, x_{s}\right)$, we get a contradiction for $C$ large enough.

The underlying reason for the second part of the proof can be explained as follows. $A$ is uniform: most of its sections (in a given direction) have approximately the same size. (The same is true for projections.) Therefore, if $U$ is some part of $A$ that has small sections in some direction, \#U is small compared to \#A and such $U$ 's cannot cover $A$.

\section{Prefix complexity}

All inequalities for Kolmogorov complexities were considered up to $O(\log n)$ term, where $n$ is a sum of complexities of strings involved. Therefore we could safely ignore the difference between several existing versions of complexity. We can use plain complexity defined by Kolmogorov in [1]), denoted by $C(x)$ in [6] and $K S(x)$ in [7], or prefix complexity, denoted by $K(x)$ in [6] and $K P(x)$ in [7].

In this section we are interested in equalities valid up to $O(1)$. Therefore we should be careful and specify exactly the version of complexity we use. Most useful here is prefix complexity $K P(x)$. For example, the inequality $K P(x, y) \leq K P(x)+K P(y)+O(1)$ is well known (see [6], example 3.1.2, p. 194). The inequality $2 K P(x, y, z) \leq K P(x, y)+K P(x, z)+K P(y, z)$ was proved (using Cauchy-Schwartz inequality) in [2]. These examples make the following conjecture plausible: 
Conjecture. Any linear inequality involving unconditional complexities that is valid up to logarithmic term is valid up to $O(1)$ for prefix complexity.

A partial result in this direction:

Theorem 4 Basic inequality (11) is valid up to $O(1)$ term for prefix complexity:

$$
K P(x)+K P(x, y, z) \leq K P(x, y)+K P(x, z) .
$$

Proof. This theorem can be easily derived from L.A. Levin's formula for prefix complexity of a pair: $K P(x, y)=K P(x)+K P(y \mid x, K P(x)$ ) (for the proof see, e.g., [6], theorem 3.9.1, p. 232). Indeed, this formula allows us to rewrite (19) as

$$
\begin{aligned}
& K P(y, z \mid x, K P(x)) \leq \\
& \quad \leq K P(y \mid x, K P(x))+K P(z \mid x, K P(x)),
\end{aligned}
$$

and this inequality is a "relativized" version of the inequality $K P(y, z) \leq K P(y)+K P(z)$.

We provide also a direct proof of (19) using a priori probabilities. Recall that $K P(x)=-\log \mathbf{m}(x)$, where $\mathbf{m}$ is universal enumerable semimeasure (see [6], p. 247). Therefore, we need to prove that

$$
\mathbf{m}(x, y, z) \mathbf{m}(x) \geq \mathbf{m}(x, y) \mathbf{m}(x, z) .
$$

or

$$
\mathbf{m}(x, y, z) \geq \frac{\mathbf{m}(x, y) \mathbf{m}(x, z)}{\mathbf{m}(x)} .
$$

Since $\sum_{y} \mathbf{m}(x, y) \leq \mathbf{m}(x)$ and $\sum_{z} \mathbf{m}(x, z) \leq \mathbf{m}(x)$, we conclude that

$$
\sum_{x, y, z} \frac{\mathbf{m}(x, y) \mathbf{m}(x, z)}{\mathbf{m}(x)} \leq \sum_{x} \mathbf{m}(x)<1 .
$$

(In fact we know only that $\sum_{y} \mathbf{m}(x, y)=O(\mathbf{m}(x))$ and $K P(x)=-\log \mathbf{m}(x)+O(1)$, but for simplicity we assume that $\sum_{y} \mathbf{m}(x, y) \leq \mathbf{m}(x)$ and $K P(x)=$ $-\log \mathbf{m}(x)$ and omit some constants in the proof.)

If the fraction $\mathbf{m}(x, y) \mathbf{m}(x, z) / \mathbf{m}(x)$ were enumerable from below, the proof would be complete, since $\mathbf{m}(x, y, z)$ is maximal. However, we have $\mathbf{m}$ in the denominator, and the fraction is not enumerable from below. We need to find an enumerable upper bound for this fraction having finite sum. For each $n$ by $\mathbf{m}_{n}(x, y)$ we denote the enumerable function obtained from $\mathbf{m}(x, y)$ by adding an additional requirement $\sum_{y} \mathbf{m}_{n}(x, y) \leq 2^{-n}$. (We eliminate values of $\mathbf{m}$ that can violate this requirement.) Now consider the function

$$
\sum_{n \geq K P(x)} \frac{\mathbf{m}_{n}(x, y) \mathbf{m}_{n}(x, z)}{2^{-n}}
$$

This sum is an upped bound for

$$
\frac{\mathbf{m}(x, y) \mathbf{m}(x, z)}{\mathbf{m}(x)}
$$

(let $n=K P(x)$; then $2^{-n}=\mathbf{m}(x)$ and $\mathbf{m}_{n}=\mathbf{m}$ ). It is an enumerable upper bound we asked for, since

$$
\begin{aligned}
& \sum_{x, y, z} \sum_{n \geq K P(x)} \frac{\mathbf{m}_{n}(x, y) \mathbf{m}_{n}(x, z)}{2^{-n}} \leq \\
& \leq \sum_{x} \sum_{n \geq K P(x)} \frac{\sum_{y} \mathbf{m}_{n}(x, y) \sum_{z} \mathbf{m}_{n}(x, z)}{2^{-n}} \leq \\
& \quad \leq \sum_{x} \sum_{n \geq K P(x)} 2^{-n} \leq \sum_{x} 2 \mathbf{m}(x) \leq 2 .
\end{aligned}
$$

Theorem 4 is proved.

Corollary: all inequalities involving unconditional complexities, having one term in the left-hand side and being true up to logarithmic term, are true up to $O(1)$ for prefix complexity.

(Indeed, theorem 2 guarantees that such an inequality is a positive linear combination of basic inequalities, so we can apply theorem 4.)

This corollary can be proved directly using semimeasures and the following version of Jensen's inequality: if $\alpha_{1}+\ldots+\alpha_{s}=1, \alpha_{i} \geq 0$, then

$$
\begin{aligned}
\int\left[f_{1}(x)\right]^{\alpha_{1}} & \ldots\left[f_{s}(x)\right]^{\alpha_{s}} d x \leq \\
\leq & \left.\leq \int f_{1}(x) d x\right]^{\alpha_{1}} \cdots\left[\int f_{s}(x) d x\right]^{\alpha_{s}}
\end{aligned}
$$

\section{Acknowledgements}

This work was done while visiting Ecole Normale Supériore de Lyon, Laboratoire de l'Informatique du Parallélisme.

Authors are grateful to all participants of Kolmogorov seminar (Moscow) and especially to M. Ushakov for his help in writing this paper. 


\section{References}

[1] A. N. Kolmogorov. Tri podkhoda $\mathrm{k}$ opredeleniju ponjatija "kolichestvo informatsii", Problemy peredachi informatsii, tom 1, no. 1, 1965, pp. 3-11. (Translation: Three approaches to the definition of the quantity of information. Prob. Inform. Transmission, 1(1), 1965, pp. 4-7.)

[2] D. Hammer, A. Shen. A Strange Application of Kolmogorov Complexity. Theory of Computing Systems, 31, 1998, pp. 1-4. (See also Tech. Report CS-R9328, CWI, Netherlands, 1993.)

[3] D. Hammer. Complexity inequalities. Wissenschaft \& Technik Verlag, Berlin, ISBN 3-89685-479-8, 1998. $143 \mathrm{pp}$.

[4] D. Hammer, A. Romashchenko, A. Shen, N. Vereshchagin. Inequalities for Kolmogorov complexities and Shannon entropies. Proceedings of $C^{2} C^{\prime} 97$, Ulm. (To be published in JCSS.)

[5] J. Llewellyn, J. Radhakrishnan, On Shearer's Lemma. Personal communication.

[6] M. Li, P. Vitanyi. An Introduction to Kolmogorov Complexity and Its Applications. Second edition, SpringerVerlag, 1997.

[7] V.A. Uspensky, A. Shen. Relations between varieties of Kolmogorov complexities. Math. Systems Theory, 29, 1996, pp. 271-292. 